\title{
Quantum optical spring
}

\author{
Amit Rai and G. S. Agarwal \\ Department of Physics, Oklahoma State University, Stillwater, Oklahoma 74078, USA
}

(Received 23 January 2008; published 22 July 2008)

\begin{abstract}
We study the dynamics of the quantum optical spring, i.e., a spring whose spring constant undergoes discrete jumps depending on the quantum state of another system. We show the existence of revivals and fractional revivals in the quantum dynamics reminiscent of similar dynamical features in cavity QED. We recover in the semiclassical limit the results for an oscillator whose frequency undergoes a sudden change. The quantum optical spring is conceivable, for example, by a micromirror under the influence of radiation pressure by a field which is strictly quantum mechanical. Our work suggests that driven systems would in general exhibit a very different dynamics if the drive is replaced by a quantum source.
\end{abstract}

DOI: 10.1103/PhysRevA.78.013831

PACS number(s): 42.50.Pq, 42.60.Da

\section{INTRODUCTION}

The quantum dynamics of an oscillator with timedependent modulation of its frequency has been investigated very extensively [1-4]. This is because of its many applications in different contexts. One very interesting application was in the context of center-of-mass motion of an ion in the Paul trap. More recently, the study of modulated oscillator acquired new importance in connection with BEC's where trap potential can easily be manipulated $[5,6]$. Further nanomechanical oscillators provided new impetus for the study of quantum dynamics of a frequency modulated system [7]. One of the important outcomes of such studies was that mechanical and vibrational degrees of freedom can be prepared in nonclassical states $[2,8]$. In particular, one finds that sudden changes in the frequency of the oscillator yield a significant amount of squeezing.

In all of the above studies the oscillator was treated as a quantum system whereas the source of modulation was considered as something prescribed. Clearly a complete quantum dynamical theory should consider the source also to be a quantized system. This is important as the mechanical oscillator can have significant back action on the source of modulation. The system is then a quantum optical spring, i.e., a spring whose spring constant depends on the quantum state of another system. The modulation by a quantized source produces new features in the quantum dynamics as the oscillator samples the discrete structure of the quantized source of modulation. In particular we show the appearance of characteristic quantum revivals and fractional revivals [9-11]. The dynamics we present should be realized by the effects of radiation pressure on a micromirror $[12,13]$.

The organization of the paper is as follows: In Sec. II, we describe the model and derive analytical result for the reduced density matrix of the oscillator and the source of modulation. The quantum dynamics of the oscillator is discussed in Sec. III. In Sec. IV, we analyze the squeezing properties. The back-action effect is described in Sec. V.

\section{MODEL FOR THE QUANTUM OPTICAL SPRING}

Consider a harmonic oscillator with mass $m$ and frequency $\omega$. Its Hamiltonian is given by

$$
H_{0}=\frac{p^{2}}{2 m}+\frac{m \omega^{2} x^{2}}{2},
$$

where $x$ and $p$ are the position and momentum coordinates obeying the canonical commutation relation $[x, p]=i \hbar$. Let us assume that the frequency of the oscillator is modulated by a quantized source such that the Hamiltonian in Eq. (1) goes to

$$
H=\frac{p^{2}}{2 m}+\frac{m \omega^{2}\left(1+\mu a^{\dagger} a\right) x^{2}}{2} .
$$

Since the interaction part commutes with the unperturbed Hamiltonian of the quantized source we do not write this part of the energy. Further for brevity we would denote by QSM as the quantized source of modulation. The operators $a$ and $a^{\dagger}$ for the QSM obey the Boson commutation relations $\left[a, a^{\dagger}\right]=1$. The Hamiltonian (2) describes the quantum optical spring. Let the eigenstates and eigenvalues $E_{n}^{(0)}$ of $H_{0}$ be denoted by

$$
\begin{gathered}
\phi_{n}(x)=N_{n} H_{n}(\alpha x) \exp \left(-\frac{1}{2} \alpha^{2} x^{2}\right), \\
E_{n}^{(0)}=\hbar \omega\left(n+\frac{1}{2}\right), \\
N_{n}=\left(\frac{\alpha}{\sqrt{\pi} 2^{n} n !}\right)^{1 / 2}, \\
\alpha \equiv\left(\frac{m \omega}{\hbar}\right)^{1 / 2},
\end{gathered}
$$

where $H_{n}$ is the $n$th Hermite polynomial.

The eigenstates of $H$ are given by

$$
\begin{gathered}
a^{\dagger} a|p\rangle=p|p\rangle, \\
H \psi_{n}^{p}|p\rangle=E_{n}^{(p)} \psi_{n}^{p}|p\rangle, \\
E_{n}^{(p)}=\hbar \omega_{p}\left(n+\frac{1}{2}\right),
\end{gathered}
$$




$$
\omega_{p} \equiv \omega \sqrt{(1+\mu p)} .
$$

and where the wave function $\psi_{n}^{p}$ is given by

$$
\begin{gathered}
\psi_{n}^{p}=N_{n} H_{n}\left(\alpha_{p} x\right)\left(\sqrt{\frac{\alpha_{p}}{\alpha}}\right) \exp \left(-\frac{1}{2} \alpha_{p}^{2} x^{2}\right), \\
\alpha_{p} \equiv\left(\frac{m \omega_{p}}{\hbar}\right)^{1 / 2} .
\end{gathered}
$$

Note that $\psi_{n}^{p}$ is the eigenstate of harmonic oscillator with frequency $\omega$ replaced by $\omega_{p}$. For a fix $p$, these form a complete set.

The time evolution of the initial state can be obtained from the knowledge of the states given by Eq. (4). Let us consider the initial state of the coupled system given by

$$
\psi(t=0)=\sum_{p, n} C_{p n} \phi_{n}(x)|p\rangle,
$$

where $C_{p n}$ are the expansion coefficients. The state at time $t$ would then be

$$
\begin{aligned}
|\psi(t)\rangle & =\exp \left(\frac{-i H t}{\hbar}\right) \psi(t=0) \\
& =\sum C_{p n}|p\rangle \exp \left[\frac{-i t}{\hbar}\left(\frac{p^{2}}{2 m}+\frac{m \omega_{p}^{2} x^{2}}{2}\right)\right] \phi_{n}(x) \\
& =\sum C_{p n}|p\rangle \exp \left[\frac{-i t}{\hbar}\left(\frac{p^{2}}{2 m}+\frac{m \omega_{p}^{2} x^{2}}{2}\right)\right] \sum_{l}\left|\psi_{l}^{p}\right\rangle\left\langle\psi_{l}^{p} \mid \phi_{n}\right\rangle \\
& =\sum_{p, n, l} C_{p n} \exp \left(\frac{-i t E_{l}^{p}}{\hbar}\right)\left\langle\psi_{l}^{p} \mid \phi_{n}\right\rangle|p\rangle\left|\psi_{l}^{p}\right\rangle .
\end{aligned}
$$

This is the state of the combined system of oscillator and the QSM. It is a nonfactorized state and thus the spring gets entangled to the quantum system controlling the spring constant. The reduced state of the oscillator and the QSM can be obtained by projecting out the degrees of freedom of the other system. It is seen that the density matrix for the oscillator is

$$
\begin{aligned}
\rho_{o}= & \sum_{n, l, m, j, p}\left|\psi_{l}^{p}\right\rangle\left\langle\psi_{j}^{p}\right| C_{p n} C_{p m}^{*} \exp \left(\frac{-i t\left(E_{l}^{p}-E_{j}^{p}\right)}{\hbar}\right)\left\langle\psi_{l}^{p} \mid \phi_{n}\right\rangle \\
& \times\left\langle\phi_{m} \mid \psi_{j}^{p}\right\rangle,
\end{aligned}
$$

and that for the QSM is

$$
\begin{gathered}
\rho_{\mathrm{QSM}}=\sum_{p_{1}, p_{2}} d_{p_{1} p_{2}}\left|p_{1}\right\rangle\left\langle p_{2}\right|, \\
d_{p_{1} p_{2}}=\sum_{n, l, m, j} C_{p_{1} n} C_{p_{2} m}^{*}\left\langle\psi_{j}^{p_{2}} \mid \psi_{l}^{p_{1}}\right\rangle\left\langle\psi_{l}^{p_{1}} \mid \phi_{n}\right\rangle \\
\times\left\langle\phi_{m} \mid \psi_{j}^{p_{2}}\right\rangle \exp \left(\frac{-i t\left(E_{l}^{p_{1}}-E_{j}^{p_{2}}\right)}{\hbar}\right) .
\end{gathered}
$$

Note especially the rather involved form of the reduced density matrix for the QSM. This is because the scalar product of the wave functions $\psi_{l}^{p}$ for different $p$ is nonzero,

$$
\left\langle\psi_{j}^{p_{2}} \mid \psi_{l}^{p_{1}}\right\rangle \neq 0 \quad \text { for } p_{1} \neq p_{2}
$$

$$
=\delta_{l j} \quad \text { if } p_{1}=p_{2} \text {. }
$$

Using Eq. (8) we can study the details of the quantum dynamics of the oscillator coupled to the QSM. We would like to mention that the quantum characteristics of a nanomechanical oscillator coupled to a quantized photon source has been studied by several authors [14-18] who have shown how nonclassical states like Schrödinger cat state of such a system can be generated. (Note that the Schrödinger cat state is the state formed from the quantum superposition of two coherent states differing in phase.) The Hamiltonian used by previous authors is given by $a^{\dagger} a x$ which is linear in the oscillator variable and is different from $a^{\dagger} a x^{2}$ which is produced by modulation. It may be noted that Arcizet et al. [12] have used precisely this Hamiltonian as they argue that the radiation pressure leads to a force which is proportional to the displacement of the oscillator. This clearly would imply an effective Hamiltonian that is quadratic in oscillator's displacement.

There are other possibilities for producing Hamiltonian (2). One would be a particle confined to a harmonic trapping potential. Its interaction with a detuned field $\vec{E}$ would produce, on account of ac Stark effect, the interaction Hamiltonian $|\vec{E}|^{2} \sin ^{2}(k x)$ which for a deep trap and in the limit of quantized field would yield the $a^{\dagger} a x^{2}$ term. Another possibility is in connection with second-order Raman scattering involving two phonons which is well known [8] to yield a Hamiltonian of the form $|E|^{2} x^{2}$ where $x$ is the displacement associated with phonons.

\section{QUANTUM DYNAMICS OF THE QUANTUM OPTICAL SPRING: REVIVALS AND FRACTIONAL REVIVALS}

Let us consider first a simple case when the QSM is prepared in a coherent state with mean amplitude $\alpha$ and the oscillator is prepared in its ground state. Thus the coefficients $C_{p n}$ are given by

$$
C_{p n}=\delta_{n 0} \frac{\alpha^{p} \exp \left(\frac{-|\alpha|^{2}}{2}\right)}{\sqrt{(p) !}} .
$$

Using Eq. (12) in Eq. (8) and on simplification we obtain

$$
\begin{aligned}
\rho_{o}= & \sum \frac{|\alpha|^{2 p} \exp \left(-|\alpha|^{2}\right)}{(p) !} \exp \left[-i t \omega_{p}(l-j)\right]\left|\psi_{l}^{p}\right\rangle\left\langle\psi_{j}^{p}\right|\left\langle\psi_{l}^{p} \mid \phi_{0}\right\rangle \\
& \times\left\langle\phi_{0} \mid \psi_{j}^{p}\right\rangle .
\end{aligned}
$$

In particular the probability of finding the oscillator in the initial state is given by 


$$
\begin{aligned}
P_{0}(t)= & \left\langle\phi_{0}\left|\rho_{o}\right| \phi_{0}\right\rangle \\
= & \sum_{p}\left(\left.\left|\sum_{l} \exp \left(-i t \omega_{p} l\right)\right|\left\langle\psi_{l}^{p} \mid \phi_{0}\right\rangle\right|^{2} \mid\right)^{2} \\
& \times\left(\frac{|\alpha|^{2 p} \exp \left(-|\alpha|^{2}\right)}{(p) !}\right)=\sum_{p}\left|A_{p}\right|^{2} \frac{|\alpha|^{2 p} \exp \left(-|\alpha|^{2}\right)}{(p) !},
\end{aligned}
$$

where

$$
A_{p}=\sum_{l} \exp \left(-i t \omega_{p} l\right)\left|\left\langle\psi_{l}^{p} \mid \phi_{0}\right\rangle\right|^{2} .
$$

This is one of our key results. The quantum state of the source appears through the weight factor in Eq. (14) and through the discrete sum in Eq. (14). Such sums have been encountered before in connection with the cavity QED problems $[9,10]$ and have been experimentally studied $[19,20]$. The sum in Eq. (15) can be evaluated in closed form since

$$
\begin{aligned}
\left\langle\psi_{l}^{p} \mid \phi_{0}\right\rangle= & \int \psi_{l}^{p}(x) \phi_{0}(x) d x \\
= & N_{l} N_{0}\left(\sqrt{\frac{\alpha_{p}}{\alpha}}\right) \int H_{l}\left(\alpha_{p} x\right) \\
& \times \exp \left[\left(-\frac{1}{2}\left(\alpha_{p}^{2}+\alpha^{2}\right) x^{2}\right)\right] d x \\
= & N_{l} N_{0}\left(\frac{\beta_{p}}{\sqrt{\alpha_{p} \alpha}}\right) \int H_{l}\left(\beta_{p} y\right) \exp \left(-y^{2}\right) d y \\
= & N_{2 m} N_{0}\left(\frac{\beta_{p}}{\sqrt{\alpha_{p} \alpha}}\right)\left(\frac{\sqrt{\pi}(2 m) !\left(\beta_{p}^{2}-1\right)^{m}}{(m) !}\right) \\
= & \frac{\beta_{p}}{\left(\eta_{p}\right)^{1 / 8}}\left(\frac{\sqrt{(2 m) !}\left(\beta_{p}^{2}-1\right)^{m}}{2^{m}(m) !}\right),
\end{aligned}
$$

where $l$ has been set equal to $2 m, m=0,1,2, \ldots$, as the scalar product is zero for odd $l$. Further, in Eq. (16) we have defined

$$
\begin{gathered}
\eta_{p}=(1+\mu p), \\
\beta_{p}^{2}=\frac{2\left(\eta_{p}\right)^{1 / 2}}{1+\left(\eta_{p}\right)^{1 / 2}} .
\end{gathered}
$$

On using Eq. (16) in Eq. (15) we find the closed form expression for $A_{p}$,

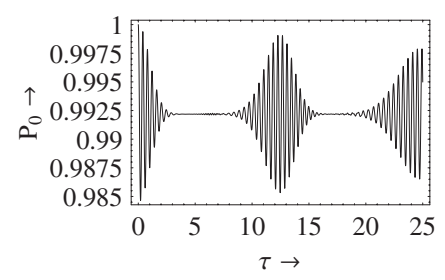

FIG. 1. The variation of $P_{0}$ as a function of $\tau(\omega t=2 \pi \tau)$ with parameter $\mu=0.1$ is shown. The average number $|\alpha|^{2}$ of excitation in the QSM is 4.

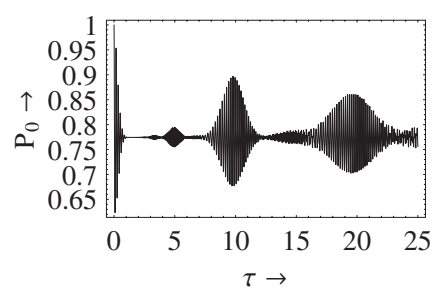

FIG. 2. The variation of $P_{0}$ as a function of $\tau(\omega t=2 \pi \tau)$ with parameter $\mu=0.3$ is shown. The average number $|\alpha|^{2}$ of excitation in the QSM is 25 .

$$
A_{p}=\frac{\left|\beta_{p}\right|^{2}}{\eta_{p}^{1 / 4}\left[1-\left(\beta_{p}^{2}-1\right)^{2} \exp \left(-2 i \omega_{p} t\right)\right]^{1 / 2}},
$$

and $P_{0}(t)$ can be written as

$$
P_{0}(t)=\left\langle\left|A_{p}\right|^{2}\right\rangle,
$$

and where $\langle\cdots\rangle$ is the average over the distribution of $p$.

For the classical source of modulation the corresponding result would be $\left|A_{p}\right|^{2}$ with $p$ replaced by the strength of modulation $|\alpha|^{2}$,

$$
\begin{gathered}
P_{c l}(t)=\frac{\left|\beta_{\alpha}\right|^{4}}{\sqrt{\eta_{\alpha}\left[1-2\left(\beta_{\alpha}^{2}-1\right)^{2} \cos \left(2 \omega_{\alpha} t\right)+\left(\beta_{\alpha}^{2}-1\right)^{4}\right]}}, \\
\eta_{\alpha}=\left(1+\mu|\alpha|^{2}\right), \\
\beta_{\alpha}^{2}=\frac{2\left(\eta_{\alpha}\right)^{1 / 2}}{1+\left(\eta_{\alpha}\right)^{1 / 2}}, \\
\omega_{\alpha}=\omega \sqrt{\left(1+\mu|\alpha|^{2}\right)} .
\end{gathered}
$$

Thus $P_{c l}$ oscillates at the frequency $2 \omega_{\alpha}$. However, the probability $P_{0}(t)$ for the QSM behaves quite differently as shown in the Fig. 1 and exhibits collapse and revival of the classical periodic motion. In a different parameter regime the fractional revivals in $P_{0}(t)$ are seen in Fig. 2. Clearly the dynamics of the quantum optical spring is very sensitive to the quantized source of modulation. Finally as is known the dynamical behavior is sensitive to the quantum statistics of the QSM. We show in Fig. 3 the behavior of the oscillator for thermal excitation of the QSM which would, for example, be relevant in microwave cavities at low temperatures. The collapses and revivals, unlike the case of the Jaynes-Cummings model [21], are more pronounced for the present model.

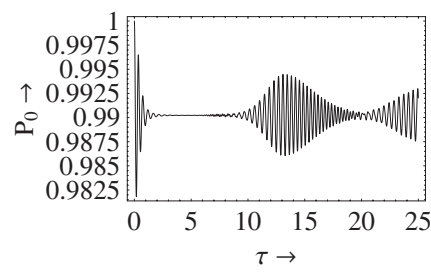

FIG. 3. The variation of $P_{0}$ as a function of $\tau(\omega t=2 \pi \tau)$ with parameter $\mu=0.1$ is shown. The average number of thermal excitation in the QSM is 4 . 


\section{SQUEEZING PROPERTIES OF QUANTUM OPTICAL SPRING}

We next investigate if the oscillator system acquires important nonclassical character as a result of the QSM. As mentioned earlier even a classical change in the frequency can lead to squeezing in oscillator and therefore an interesting question would be how this squeezing character is modified by the QSM. In order to calculate squeezing it is more convenient to work with Heisenberg equations of motion. For the Hamiltonian in Eq. (2) we can prove that

$$
\begin{gathered}
\frac{d\left(a^{\dagger} a\right)}{d t}=0, \\
\frac{d x}{d t}=\frac{p}{m}, \\
\frac{d p}{d t}=-m(\omega)^{2}\left(1+\mu a^{\dagger} a\right) x .
\end{gathered}
$$

Thus $a^{\dagger} a$ is a constant of motion and we can write the solution as

$$
\begin{gathered}
x(t)=x(0) \cos (\hat{\Omega} t)+\frac{p(0)}{m \hat{\Omega}} \sin (\hat{\Omega} t), \\
p(t)=p(0) \cos (\hat{\Omega} t)-m \hat{\Omega} x(0) \sin (\hat{\Omega} t),
\end{gathered}
$$

where $\hat{\Omega}$ is given by

$$
\hat{\Omega}=\omega \sqrt{1+\mu a^{\dagger} a}
$$

and where $a^{\dagger} a$ is the operator at time $t=0$.

We define the squeezing variables

$$
\begin{aligned}
& V_{x}(t)=\frac{\left\langle x^{2}(t)\right\rangle-\langle x(t)\rangle^{2}}{\left\langle x^{2}(0)\right\rangle}, \\
& V_{p}(t)=\frac{\left\langle p^{2}(t)\right\rangle-\langle p(t)\rangle^{2}}{\left\langle p^{2}(0)\right\rangle} .
\end{aligned}
$$

For the ground state of the oscillator

$$
\begin{gathered}
\langle x(0)\rangle=\langle p(0)\rangle=0, \quad\langle x p+p x\rangle=0, \\
\left\langle x^{2}(0)\right\rangle=\frac{\hbar}{2 m \omega}, \quad\left\langle p^{2}(0)\right\rangle=\frac{m \hbar \omega}{2} .
\end{gathered}
$$

Using Eqs. (24) and (27) we obtain

$$
V_{x}(t)=1-\sum_{n} \frac{|\alpha|^{2 n} \exp \left(-|\alpha|^{2}\right) \sin ^{2}(\omega t \sqrt{1+\mu n})}{n !}\left(\frac{\mu n}{1+\mu n}\right),
$$

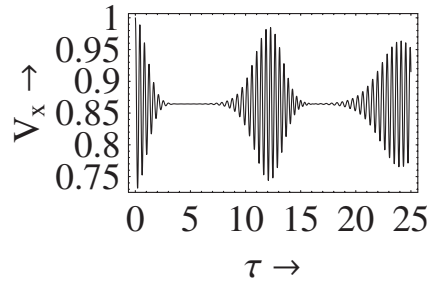

FIG. 4. The variation of $V_{x}(t)$ as a function of $\tau(\omega t=2 \pi \tau)$ with parameter $\mu=0.1$ is shown. The average number $|\alpha|^{2}$ of excitation in the QSM is 4.

$$
V_{p}(t)=1+\sum_{n} \frac{|\alpha|^{2 n} \exp \left(-|\alpha|^{2}\right) \sin ^{2}(\omega t \sqrt{1+\mu n})(\mu n)}{n !}
$$

Clearly $V_{x}(t)$ is always less than 1 and hence the $x$ quadrature is squeezed. The corresponding result with a classical source of modulation would be

$$
V_{x}(t)=1-\sin ^{2}\left(\omega t \sqrt{1+\mu|\alpha|^{2}}\right)\left(\frac{\mu|\alpha|^{2}}{1+\mu|\alpha|^{2}}\right) .
$$

The minimum value of $V_{x}$ in the classical case is when $\sin ^{2}\left(\omega t \sqrt{1+\mu|\alpha|^{2}}\right)=1$ and hence

$$
V_{x, \min }=1-\left(\frac{\mu|\alpha|^{2}}{1+\mu|\alpha|^{2}}\right) .
$$

For the QSM the squeezing parameter (28) exhibits typical collapse and revival of the classical periodical oscillation (Figs. 4 and 5). This is again due to the discrete nature of the quantum state of the source of modulation, i.e., control of the spring constant by a quantum source. We also note that under the approximation $\mu n /(1+\mu n) \simeq 1$, the sum in (28) is identical to that appearing in the Jaynes-Cummings model [10] and can be simplified by using approximate methods developed there.

\section{BACK REACTION OF THE OSCILLATOR ON THE QSM}

In this section we evaluate the back action of the oscillator on the QSM. The Hamiltonian in Eq. (2) depends only on $a^{\dagger} a$ and therefore only the off-diagonal elements of the density matrix $\rho_{\mathrm{QSM}}$ are affected by the coupling of the oscillator to the QSM. The reduced state of the QSM is rather in-

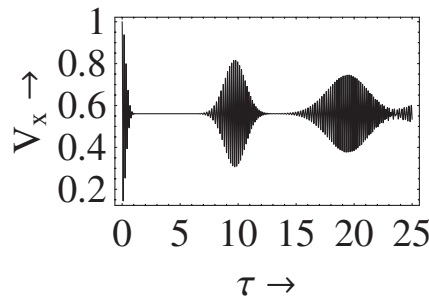

FIG. 5. The variation of $V_{x}(t)$ as a function of $\tau(\omega t=2 \pi \tau)$ with parameter $\mu=0.3$ is shown. The average number $|\alpha|^{2}$ of excitation in the QSM is 25 . 
volved. In order to appreciate the back action we examine the mean displacement $\langle\hat{a}\rangle$ of the QSM which is conditional on the measurement of oscillator in the ground state $\left|\phi_{0}\right\rangle$. The mean value is given by

$$
d(t) \equiv\langle\hat{a}\rangle=\sum_{n, l}\langle l|a| n\rangle\langle n|\rho| l\rangle=\sum_{n} \sqrt{n} \rho_{n, n-1} .
$$

We assume that the oscillator was initially in the state $\left|\phi_{0}\right\rangle$ and the QSM is in the state $|\alpha\rangle$. Then, the QSM's conditional density matrix is found to be

$$
\rho_{n, l}=c_{n}^{*} c_{l} X_{n, l}
$$

where $c_{n}$ 's are given by

$$
c_{n}=\frac{\alpha^{n} \exp \left(\frac{-|\alpha|^{2}}{2}\right)}{\sqrt{(n) !}},
$$

and $X_{n, l}$ is given by

$$
X_{n, l}=\left\langle\phi_{0}|\exp [i h(n)] \exp [-i h(l)]| \phi_{0}\right\rangle .
$$

The coefficient $X_{n, l}$ is the modification of the off-diagonal element of the density matrix of the QSM as a result of back action of the oscillator.

In the above equation we have introduced the Hamiltonian $h(n)$ defined by

$$
h(n) \equiv \frac{t}{\hbar}\left(\frac{p^{2}}{2 m}+\frac{m \omega^{2}(1+\mu n) x^{2}}{2}\right) .
$$

The $X_{n, l}$ 's can be calculated by using operator disentangling theorems for the $\mathrm{SU}(1,1)$ group. We give details in the Appendix. We find the following result [Eq. (A13)]:

$$
X_{n, l}=\frac{\left[\left(\Gamma_{3 n}\right)^{*} \Gamma_{3 l}\right]^{1 / 4}}{\sqrt{1-\left(\Gamma_{+n}\right)^{*} \Gamma_{+l}}} .
$$

The displacement of the QSM with $\Gamma$ 's defined by Eqs. (A9) and (A10) can be obtained from Eqs. (32) and (33). The coefficient $X_{n, l}$ turns out to be related to the scalar product of two squeezed vacuum states defined by

$$
\begin{aligned}
|z\rangle & \equiv \frac{1}{\sqrt{\cosh |z|}} \sum_{m=0}^{\infty}\left(\frac{\sqrt{(2 m) !}}{2^{m} m !}\right)\left(\frac{z}{|z|}\right)^{m}[\tanh (|z|)]^{m}|2 m\rangle \\
& \equiv \frac{1}{\sqrt{\cosh |z|}} \exp \left[\left(\frac{z}{|z|}\right) \tanh (|z|) \frac{\left(\hat{b}^{\dagger}\right)^{2}}{2}\right]\left|\phi_{0}\right\rangle,
\end{aligned}
$$

Thus on comparison with (A12) we find

$$
\begin{gathered}
\left(\frac{z_{n}}{\left|z_{n}\right|}\right) \equiv \frac{\Gamma_{+n}}{\left|\Gamma_{+n}\right|}, \\
\tanh \left(\left|z_{n}\right|\right)=\left|\Gamma_{+n}\right| .
\end{gathered}
$$

Thus $X_{n, l}$ can be written as

$$
X_{n, l} \equiv\left\langle z_{n} \mid z_{l}\right\rangle \text {. }
$$

In Fig. 6 we display the time evolution of the imaginary part of $\langle\hat{a}\rangle$. Note that the imaginary part of $\langle\hat{a}\rangle$ builds up starting

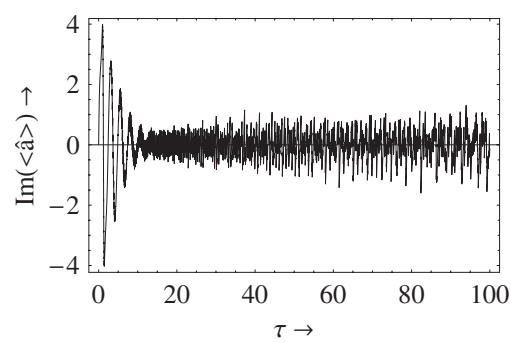

FIG. 6. The variation of the imaginary part of $\langle\hat{a}\rangle$ as a function of $\tau(\omega t=16 \pi \tau)$ with parameter $\mu=0.3$ is shown. The average number $|\alpha|^{2}$ of excitation in the QSM is 25 .

from zero and exhibits aperiodic behavior. The quantized nature of the optical spring is well reflected in the back reaction. Evidently the back reaction occurs over a much larger time scale.

In conclusion we introduced the idea of a quantum optical spring, i.e., a spring whose spring constant is controlled by another quantum system. The dynamics of the oscillator system exhibits the phenomena of collapse and revivals including fractional revivals. Further the spring gets entangled to the quantum system which controls the spring constant.

\section{APPENDIX: CALCULATION OF THE MATRIX ELEMENT Eq. (35)}

In the appendix we give the details of our calculation for $X_{n l}$. It is convenient to use $x$ and $p$ in terms of annihilation and creation operators $\hat{b}$ and $\hat{b}^{\dagger}$,

$$
\begin{aligned}
& x=\sqrt{\frac{\hbar}{2 m \omega}}\left(\hat{b}^{\dagger}+\hat{b}\right), \\
& p=i \sqrt{\frac{\hbar m \omega}{2}}\left(\hat{b}^{\dagger}-\hat{b}\right), \\
& \exp [-i h(n)]\left|\phi_{0}\right\rangle=\exp \left[-\frac{i t}{\hbar}\left(\frac{p^{2}}{2 m}+\frac{m \omega^{2}(1+\mu n) x^{2}}{2}\right)\right]\left|\phi_{0}\right\rangle,
\end{aligned}
$$

Substituting the values for $x$ and $p$ from Eq. (A1) in Eq. (A2) and on simplification we obtain

$$
\exp [-i h(n)]\left|\phi_{0}\right\rangle=\exp \left(\gamma_{+n} \hat{K}_{+}+\gamma_{-n} \hat{K}_{-}+\gamma_{3 n} \hat{K}_{3}\right)\left|\phi_{0}\right\rangle,
$$

where

$$
\begin{gathered}
\gamma_{ \pm n} \equiv \frac{-i \mu \omega n t}{2}, \\
\gamma_{3 n} \equiv-i \omega t(\mu n+2) .
\end{gathered}
$$

In (A3) $\hat{K}_{ \pm}, \hat{K}_{3}$ are the generators of the $\mathrm{SU}(1,1)$ algebra given by

$$
\hat{K}_{3} \equiv \frac{1}{4}\left(\hat{b}^{\dagger} \hat{b}+\hat{b} \hat{b}^{\dagger}\right)
$$




$$
\hat{K}_{+}=\left(\hat{K}_{-}\right)^{\dagger}=\frac{1}{2}\left(\hat{b}^{\dagger}\right)^{2}
$$

Equation (A3) can be simplified by using the disentangling theorem [22] for the $\mathrm{SU}(1,1)$ group,

$$
\begin{aligned}
& \exp \left(\gamma_{+n} \hat{K}_{+}+\gamma_{-n} \hat{K}_{-}+\gamma_{3 n} \hat{K}_{3}\right) \\
& \quad=\exp \left(\Gamma_{+n} \hat{K}_{+}\right) \exp \left[\left(\ln \Gamma_{3 n}\right) \hat{K}_{3}\right] \exp \left(\Gamma_{-n} \hat{K}_{-}\right),
\end{aligned}
$$

where

$$
\begin{gathered}
\Gamma_{3 n} \equiv \frac{1}{\left(\cosh \lambda_{n}-\frac{\gamma_{3 n}}{2 \lambda_{n}} \sinh \lambda_{n}\right)^{2}}, \\
\Gamma_{ \pm n} \equiv \frac{2 \gamma_{ \pm n} \sinh \lambda_{n}}{\left(2 \lambda_{n} \cosh \lambda_{n}-\gamma_{3 n} \sinh \lambda_{n}\right)}, \\
\lambda_{n}^{2} \equiv \frac{1}{4} \gamma_{3 n}^{2}-\gamma_{+n} \gamma_{-n} .
\end{gathered}
$$

We use the disentangling theorem in the form (A8) as then $\hat{K}_{-}$acting on $\left|\phi_{0}\right\rangle$ yields zero and $\hat{K}_{3}$ terms can be simplified as $\left|\phi_{0}\right\rangle$ is an eigenstate of $\hat{K}_{3}$. Thus we reduce Eq. (A3) to

$$
\begin{aligned}
& \exp \left(\gamma_{+n} \hat{K}_{+}+\gamma_{-n} \hat{K}_{-}+\gamma_{3 n} \hat{K}_{3}\right)\left|\phi_{0}\right\rangle \\
& \quad \equiv \exp \left(\Gamma_{+n} \hat{K}_{+}\right) \exp \left[\left(\ln \Gamma_{3 n}\right) \hat{K}_{3}\right] \exp \left(\Gamma_{-n} \hat{K}_{-}\right)\left|\phi_{0}\right\rangle \\
& \quad=\exp \left(\frac{\left(\ln \Gamma_{3 n}\right)}{4}\right) \exp \left(\Gamma_{+n} \hat{K}_{+}\right)\left|\phi_{0}\right\rangle \\
& \quad=\left(\Gamma_{3 n}\right)^{1 / 4} \exp \left(\Gamma_{+n} \hat{K}_{+}\right)\left|\phi_{0}\right\rangle .
\end{aligned}
$$

Hence $X_{n, l}$ as defined by (35) can be calculated as follows:

$$
\begin{aligned}
X_{n, l} & =\left\langle\phi_{0}|\exp [i h(n)] \exp [-i h(l)]| \phi_{0}\right\rangle \\
& =\left[\left(\Gamma_{3 n}\right)^{*} \Gamma_{3 l}\right]^{1 / 4}\left\langle\phi_{0}\left|\exp \left[\left(\Gamma_{+n}\right)^{*} \hat{K}_{-}\right] \exp \left(\Gamma_{+l} \hat{K}_{+}\right)\right| \phi_{0}\right\rangle \\
& =\left[\left(\Gamma_{3 n}\right)^{*} \Gamma_{3 l}\right]^{1 / 4} \sum_{p} \frac{\left[\left(\Gamma_{+n}\right)^{*} \Gamma_{+l}\right]^{p}(2 p) !}{(p !)^{2} 2^{2 p}}=\frac{\left[\left(\Gamma_{3 n}\right)^{*} \Gamma_{3 l}\right]^{1 / 4}}{\sqrt{1-\left(\Gamma_{+n}\right)^{*} \Gamma_{+l}}} .
\end{aligned}
$$

This is the result we use in Sec. V to calculate the back action.
[1] J. Janszky and Y. Y. Yushin, Opt. Commun. 59, 151 (1986).

[2] G. S. Agarwal and S. A. Kumar, Phys. Rev. Lett. 67, 3665 (1991).

[3] R. Graham, J. Mod. Opt. 34, 873 (1987).

[4] R. J. Glauber, in Quantum Optics, edited by R. Inguva (Plenum, New York, 1991).

[5] G. Behinaein, V. Ramareddy, P. Ahmadi, and G. S. Summy, Phys. Rev. Lett. 97, 244101 (2006).

[6] S. Choi and N. P. Bigelow, J. Mod. Opt. 52, 1081 (2005).

[7] M. P. Blencowe and M. N. Wybourne, Physica B (Amsterdam) 280, 555 (2000).

[8] G. A. Garrett, A. G. Rojo, A. K. Sood, J. F. Whitaker, and R. Merlin, Science 275, 1638 (1997).

[9] J. H. Eberly, N. B. Narozhny, and J. J. Sanchez-Mondragon, Phys. Rev. Lett. 44, 1323 (1980).

[10] W. P. Schleich, Quantum Optics in Phase Space (Wiley, New York, 2001).

[11] C. Leichtle, I. Sh. Averbukh, and W. P. Schleich, Phys. Rev. A 54, 5299 (1996); D. L. Aronstein and C. R. Stroud, ibid. 55, 4526 (1997); Z. D. Gaeta and C. R. Stroud, Jr., ibid. 42, 6308 (1990).

[12] O. Arcizet, P. F. Cohadon, T. Briant, M. Pinard, and A. Heidmann, Nature (London) 444, 71 (2006).

[13] We do not include damping effects in the analysis which are known to be detrimental for the observation of such revival effects. These could be incorporated by using the known methods: See, for example, S. M. Barnett and P. L. Knight, Phys. Rev. A 33, 2444 (1986); R. R. Puri and G. S. Agarwal, ibid. 33, 3610 (1986).

[14] W. Marshall, C. Simon, R. Penrose, and D. Bouwmeester, Phys. Rev. Lett. 91, 130401 (2003).

[15] S. Bose, K. Jacobs, and P. L. Knight, Phys. Rev. A 59, 3204 (1999).

[16] A. D. Armour, M. P. Blencowe, and K. C. Schwab, Phys. Rev. Lett. 88, 148301 (2002).

[17] C. K. Law and J. H. Eberly, Phys. Rev. A 43, 6337 (1991).

[18] A. Naik et al., Nature (London) 443, 193 (2006); S. Gigan et al., ibid. 444, 67 (2006); D. Kleckner and D. Bouwmeester, ibid. 444, 75 (2006).

[19] J. M. Raimond, M. Brune, and S. Haroche, Rev. Mod. Phys. 73, 565 (2001).

[20] H. Walther, B. T. H. Varcoe, B. G. Englert, and T. Becker, Rep. Prog. Phys. 69, 1325 (2006).

[21] P. L. Knight and P. M. Radmore, Phys. Lett. 90A, 342 (1982).

[22] S. M. Barnett and P. M. Radmore, Methods in Theoretical Quantum Optics (Oxford University Press, Oxford, 2002), p. 238. 